\title{
Study on adsorption characteristics and regeneration effect of iron - based alumina composites
}

\author{
Bai Sun ${ }^{1,2}$, Fangwen $\mathrm{Xu}^{1,2}$, Fei Liu ${ }^{1,2}$, Hui Wang ${ }^{1,2}$, Chengfeng Ding ${ }^{1,2}$, Yunming Cheng ${ }^{1,2}$, Zhuo Tang ${ }^{1,2}$, Jie Zhang ${ }^{1,2}$, Wei \\ Shen $^{3^{*}}$, and Shuguang $\mathrm{Zhu}^{1,2^{*}}$ \\ ${ }^{1}$ Key Laboratory of Water Pollution Control and Wastewater Resource of Anhui Province, College of Environment and Energy \\ Engineering, Anhui Jianzhu University, Hefei, 230601, China. \\ ${ }^{2}$ Key Laboratory of Huizhou Architecture in Anhui Province, Anhui Jianzhu University, Hefei, 230601, China. \\ ${ }^{3}$ Nano-materials and environmental detection laboratory, Hefei Institute of Physical Science, Chinese Academy of Sciences, Hefei, \\ 230031, China.
}

\begin{abstract}
In this paper, an iron-based alumina composite was synthesized by modifying activated alumina with ferric chloride. The adsorption performance of iron-based alumina composite modified with $15 \%$ ferric chloride solution reached a certain peak. At lower initial fluoride concentration, the amount of fluoride treated by this composite is much more than that of water samples with higher initial fluoride concentration. To some degree, the slower flow rate of fluoride solution, the better adsorption performance of adsorbent. The iron-based alumina composite expressed a better performance of fluoride adsorption at $\mathrm{pH}=6.5$. The adsorbent treated with aluminum potassium sulfate had the best adsorption performance.
\end{abstract}

\section{Introduction}

The concentration of fluoride in drinking water has a significant impact on human health. If human body uptake of fluoride in the right concentration is good for preventing tooth decay, however, too much of it would lead to tooth and bone disease. According to the standards of the World Health Organization (WHO), the appropriate concentration of fluorine ion in drinking water is $0.5-1.5 \mathrm{mg} / \mathrm{L}^{[1]}$, while the Chinese safe drinking water standard stipulates that the concentration of fluorine ion prohibited exceed $1.0 \mathrm{mg} / \mathrm{L}^{[2]}$. Fluoride-bearing groundwater is widely distributed in China, there were still about 70 million people drink excessive fluoride water, especially in northeast, north and northwest $\mathrm{China}^{[3]}$. In the past 20 or 30 years, there have been a lot of studies on the treatment of fluorine-containing water at home and abroad. At present, the removal methods of fluoride in fluorine-containing water mainly include nanofiltration ${ }^{[4,5]}$, sedimentation ${ }^{[6,}$ 7], coagulation ${ }^{[8,9]}$, electrodialysis ${ }^{[10,}$ 11], reverse osmosis $^{[12,13]}$, adsorption ${ }^{[14,15]}$ and ion exchange. Among the commonly used methods for removing fluorine, the adsorption method is one of the most widely used because of its simple operation, low cost, high selectivity and efficiency, and the wide variety of adsorbent. The activated-alumina (AA) grains are considered as spherical and porous, with constant diameter and with adsorption sites uniformly distributed throughout the grains, it was strongly showed that activated-alumina has a excellent adsorption properties. Therefore, in this paper, activated alumina was improved performance with ferric

\footnotetext{
${ }^{*}$ Corresponding author

E-mail address: 286537056@qq.com (S. G. Zhu),

vickyshen@iim.ac.cn (W. Shen).
}

chloride was used to remove fluoride, and adsorption experiments under different conditions were studied and discussed according to using and regeneration of regenerant.

\section{Materials and methods}

\subsection{Reagents and instruments}

The reagents were used in the experiments were mainly about aluminum hydroxide, potassium aluminum sulfate, sodium sulfate, sodium hydroxide, hydrochloric acid, sodium fluoride and Hexahydrate ferric chloride. The above reagents were analytical pure and Sinopharm group chemical reagent co. LTD. The instruments were used in the experiments mainly include AL-204 balance, multi-function constant temperature and speed magnetic stirrer, low-speed large-capacity multi-tube centrifuge, electric constant temperature drum box, fluorine ion selective electrode, PHS-3C pH meter, automatic specific surface aperture distribution analyzer and measuring straw.

\subsection{Materials preparation}

In the preparation procedure, $15 \mathrm{~g}$ of ferric chloride powder was dispersed in a $200 \mathrm{~mL}$ of beaker with $100 \mathrm{~mL}$ of distilled water to prepare $15 \%$ concentration of ferric chloride solution. Then, $10 \mathrm{~g}$ of activated alumina were added and soaked into $15 \%$ concentration of ferric chloride solution for modification. At the same time, the 
various concentrations of ferric chloride solution were prepared to obtain the optimal concentration, and the optimal soaking time was also studied.

\subsection{Adsorption experiments}

Effect of concentration of ferric chloride solution on adsorption performance. The activated alumina was modified with $0 \%, 5 \%, 10 \%, 15 \%$ and $25 \%$ concentration of ferric chloride solution respectively, the unmodified activated alumina was added for experiment, and compared with the modified activated alumina of these concentrations. The initial fluoride concentration was set as $10 \mathrm{ppm}$, the flow rate ratio was $1: 6$, and the $\mathrm{pH}$ value was 7 . The fluoride solution was adsorbed with modified adsorbents of different concentrations of ferric chloride solution. Finally, chose the optimally modified activated alumina for the rest of experiments. Effect of initial fluoride concentration, flow rate ratio, $\mathrm{pH}$ and regenerated solutions, on adsorption performance would also be considered, respectively.

\section{Results and discussion}
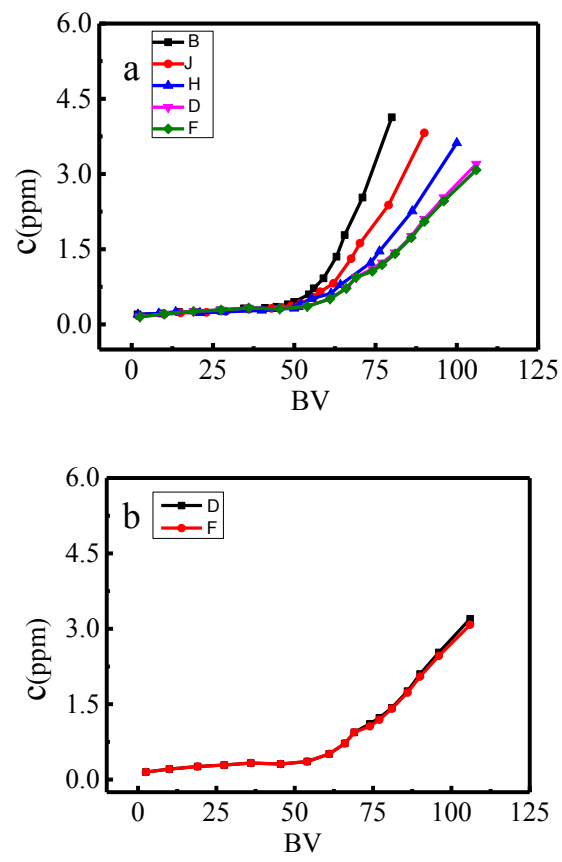

Figure 1.a. Effects of various concentrations of modifiers on fluoride removal, $b$. effects of two different concentrations of modifiers on fluoride removal effect. (Where, BV is the multiple column ratio and $\mathrm{c}$ is the effluent concentration. )

As shown in fig.1. a, the line B is unmodified activated alumina experiment to get the adsorption curve, and the line $\mathrm{J}, \mathrm{H}, \mathrm{D}$ and $\mathrm{F}$ were obtained with $5 \%, 10 \%, 15 \%$ and $25 \%$ concentration of modified activated alumina adsorption curve, respectively. In Fig. 1.b, the line D is the adsorption curve after modification with 15\% concentration, and the line $\mathrm{F}$ is the adsorption curve after modification with $25 \%$ concentration. As can be seen from the figure, the adsorption performance of activated alumina after modification with $15 \%$ concentration of iron chloride solution reached a certain peak, and the adsorption performance was better than that after modification with $0 \%, 5 \%$ and $10 \%$ concentration. However, when the concentration of $15 \%$ increased to $25 \%$, the adsorption effect did not change significantly, perhaps the modified effect of activated alumina reached saturation. Therefore, the experiment should be modified with $15 \%$ concentration of ferric chloride on activated alumina, so as to continue the experiment.

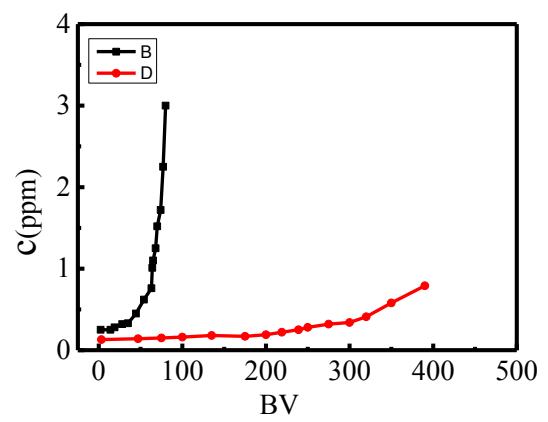

Figure 2. Effects of various initial concentrations on fluoride removal

As shown in fig. 2, both line B and D of adsorption curve were obtained when the initial fluoride concentration are $10 \mathrm{ppm}$ and $3 \mathrm{ppm}$. As can be seen from the figure, when the initial fluoride concentration was $3 \mathrm{ppm}$, the adsorption effect reached the peak at $\mathrm{BV}=80$. After that, the adsorption effect changed significantly. When the initial fluorine concentration was $10 \mathrm{ppm}$, the $\mathrm{BV}$ did not reach its peak at around 400. Comparison shows that at lower initial fluoride concentration, the amount of fluoride treated by this adsorbent is much more than the higher initial fluoride concentration, and had a better adsorption properties. This may be due to the high concentration of fluoride in the solution, and the limited ability of the adsorbent to deal with the high concentration of fluoride in a certain contact time. Therefore, in this experiment, the adsorption effect was better when the initial fluorine concentration was $3 \mathrm{ppm}$.

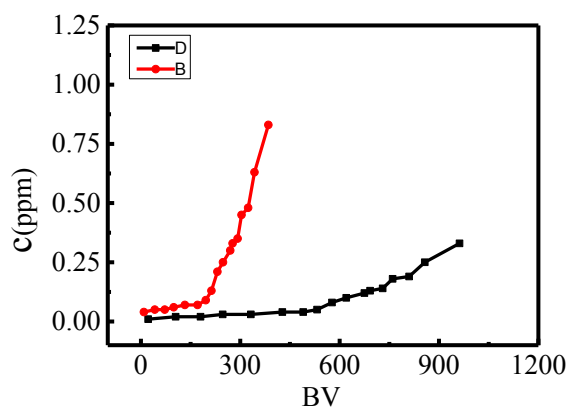

Figure 3. Effects of various flow rate ratio on fluoride removal

As shown in fig. 3, both line B and line D were obtained in the experiment when the flow rate ratio are $1: 6$ and $1: 3$. When the flow rate ratio of $1: 6$ reaches about $200 \mathrm{BV}$, the fluoride removal effect changed greatly and reached a certain peak value, while the flow rate of 1:3 did not reached the peak value when the BV 
was above 800 . It can be seen that adsorption devices with different f flow rate ratios had a great influence on the defluoride performance of adsorbent, and the flow rate of 1:3 was better than that of 1:6. This might caused by the retention time of the original fluoride solution in the adsorbent was shorter, which made the adsorbent adsorbed the fluoride solution incompletely. So the relatively slower flow rate ratio it is, the better adsorption performance would have.

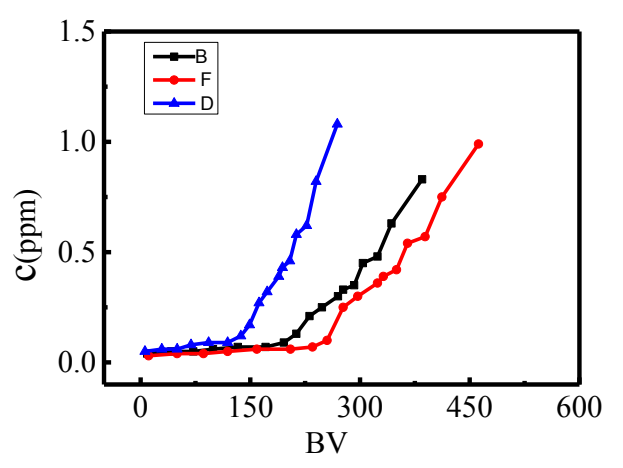

Figure 4. Effects of various $\mathrm{pH}$ on fluoride removal.

As shown in fig. 4, the line B, D and F of adsorption curve represented the effect of various $\mathrm{pH}$ on fluoride removal at 7, 6.5 and 8 , respectively. As can be seen from the figure, when $\mathrm{pH}=6.5$ and $\mathrm{BV}$ was about 150 , the adsorption performance changes greatly. When $\mathrm{pH}=7$ and BV was about 200, the adsorption effect changes greatly, and the subsequent adsorption performance did not change much. At $\mathrm{pH}=8$, the adsorption effect gradually became better when BV was about 270 . And it can be seen that the adsorption performance at $\mathrm{pH}=6.5$ were much better than that at $\mathrm{pH}$ value of 7 and 8 . It can be seen that, in the acidic environment, the modified adsorbent had a faster curve growth rate and better adsorption performance when the column ratio was smaller. Therefore, the adsorption effect of adsorbent on fluorine was better at $\mathrm{pH}=6.5$.

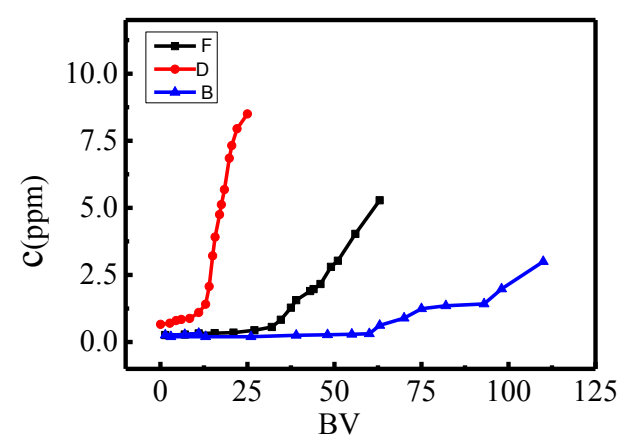

Figure 5. Effects of various regenerated liquids on fluoride removal effect.

As shown in figure 5 , the line B, D and F were the adsorption curve obtained by using the regenerated liquid for aluminum potassium sulfate, sodium hydroxide and sodium sulfate,respectively. It was obviously seen that the adsorption efficiency of aluminum potassium sulfate was optimum in the range of small column ratio. The adsorbent regenerated from aluminum potassium sulfate had the best adsorption performance, followed by sodium sulfate and sodium hydroxide. Therefore, aluminum potassium sulfate with a mass ratio of $5 \%$ should be selected as the regeneration solution for the regeneration experiment of adsorbent.

\section{Conclusions}

The adsorption effect of modified activated alumina is much better than that of unmodified activated alumina. In a particular situation, the adsorption performance of modified activated alumina was affected by many factors. Especially, the initial fluorine concentration, the flow rate ratio, the type of regenerant, the concentration of regenerant, $\mathrm{pH}$ and so on all had a great effect on its adsorption performance.

\section{Acknowledgement}

This work was supported by the National Natural Science Foundation of China (61873003), the Natural Science Major Research Projects of Anhui Education Department (KJ2017ZD40, KJ2016SD14 and KJ2017ZD41), the Science and Technology Major projects of Anhui Province (16030801118, 18030801106 and 18030801104), the Scientific Research Start-up Foundation for Introduction of Talent, Anhui Jianzhu University (2016QD113).

\section{References}

1. World Health Organization, Guidelines for Drinking-Water Quality: Incorporating First Addendum Recommendations, vol. 1, third ed., World Health Organization, 20 Avenue Appia, 1211 Geneva 27, Switzerland, (2006).

2. M. L. Wang, X. W. Zhu, X. X. Cheng, Y. L. Ye, W. Q. Wang, Z. D. Gan, T. Y. Wang, H. Y. Ding, G. B. Li. J. Water Supply Drain. 43, 11 (2017).

3. L. D. Kong, J. X. He, A. Y. Wang, H. Li. J. Sci. Technol. Inform. Develop. Econo. 16, 3 (2006).

4. N. James A, D. Francis A. J. J. 88, 14 (1996).

5. J. J. Shen, A. I. Schäfer. J. Sci. Total Environ. 527-528, 10 (2015).

6. T. T. Yang, X. J. Xu. J. Chem. Ind. Eng. Pro. 380, 13 (2015)

7. H. B. Cong, F. Sun, W. J. Chen. J. Environ. Technol. 39, 17 (2017).

8. S. Dubey, M. Agarwal, A. B. Gupta. J. J. Mol. Liq. 266, 12 (2018).

9. A. R. Donovan, C. D. Adams, Y. F. Ma, C. Stephan, T. Eichholz, H. L. Shi. J. Chemosphere, 195, 11 (2018).

10. F. D. Belkada, O. Kitous, N. Drouiche, S. Aoudj, O. Bouchelaghem, N. Abdi, H. Grib, N. Mameri. J. Sep. Purif. Technol. 204, 8 (2018). 
11. S. Gmar, I. B. S. Sayadi, N. Helali, M. Tlili, M. B. Amor. J. Environ. Pro. 2, 14 (2015).

12. A. Y. Cetinkaya, L. Bilgili. J. Water Air Soil Poll, 230, 10 (2019).

13. I. Parlar, M. Hacıfazlığlu, N. Kabay, T. O. Pek, M. Yuksel. J. J. Water Pro. Eng. 29, 7 (2019).

14. Y. Z. Li, C. Zhang, Y. P. Jiang, T. J. Wang. J. Chemosphere, 200, 7 (2018).

15. D. J. Kang, X. L. Yu, M. F. Ge. J. Chem. Eng. J. 330, 8 (2017). 